\title{
A STATISTICAL THEORY OF HEAVY ATOMS: ASYMPTOTIC BEHAVIOR OF THE ENERGY AND STABILITY OF MATTER
}

\author{
HEINZ SIEDENTOP \\ Dedicated to Ari Laptev on the occasion of his septuagesimal birthday. \\ His ideas in analysis have inspired many.
}

\begin{abstract}
We give the asymptotic behavior of the ground state energy of Engel's and Dreizler's relativistic Thomas-Fermi-Weizsäcker-Dirac functional for heavy atoms for fixed ratio of the atomic number and the velocity of light. Using a variation of the lower bound, we show stability of matter.
\end{abstract}

\section{INTRODUCTION}

Heavy atoms require a relativistic description because of the extremely fast moving inner electrons. However, a statistical theory of the atom in the the spirit of Thomas [29] and Fermi $[12,13]$ yields a functional which is unbounded from below because the semi-classical relativistic Fermi energy is too weak to prevent mass from collapsing into the nucleus. (See Gombas [17, §14] and [18, Chapter III, Section 16.]. Gombas also suggested that Weizsäcker's (non-relativistic) inhomogeneity correction would solve this problem. Tomishia [30] carried this suggestion through.) Because of the same reason the relativistic generalization of the Lieb-Thirring inequality by Daubechis is not directly applicable to Chandrasekhar operators with Coulomb potentials but requires a separate treatment of the singularity. Frank and Ekholm [10] found a way circumventing this problem treating critical potentials of Schrödinger operators; later Frank et al. [14] accomplished the same for Chandrasekhar operators with Coulomb potentials. It amounts to a Thomas-Fermi functional with a potential whose critical singularity has been extracted. However, there is a drawback, namely the Thomas-Fermi constant of this functional is smaller than the classical one, i.e., we cannot expect asymptotically correct results.

Here we discuss an alternative relativistic density functional which can handle Coulomb potentials of arbitrary strength: Engel and Dreizler [11] derived a functional $\mathcal{E}_{c, Z}^{\mathrm{TFWD}}$ of the electron density $\rho$ from quantum electrodynamics (in contrast to Gombas' ad hoc procedure of adding the non-relativistic Weizsäcker correction). It is - in a certain sense - a generalization of the non-relativistic Thomas-FermiWeizsäcker-Dirac functional to the relativistic setting, a feature that it shares with the functional investigated by Lieb et [22]. However, it does not suffer from the problem that it becomes unbounded from below for heavy atoms. We will show here, that it has - unlike the functional which can be obtained from [14] - the same asymptotic behavior as the atomic quantum energy. The price to pay is the absence of a known inequality relating it to the full quantum problem. One could speculate that it might be an upper bound on the ground state energy. The way we prove the upper bound of the asymptotics might nourishes such thoughts. However, even in the non-relativistic context this is open despite numerical evidence and claims to the contrary, e.g., by March and Young [25]: the arguments given contain a gap.

Date: January 15, 2021. 
In other words, such a claim would have - even in the non-relativistic context - at best the status of a conjecture.

Engel's and Dreizler's functional is the relativistic TF functional (see Chandrasekhar [7] [in the ultrarelativistic limit] and Gombas [17, §14] for the general case) with an inhomogeneity and exchange correction different from the nonrelativistic terms but with an integrand tending pointwise to their non-relativistic analogue for large velocity of light $c$. In Hartree units it reads for atoms of atomic number $Z$ and electron density $\rho$

$$
\mathcal{E}_{c, Z}^{\mathrm{TFWD}}(\rho):=\mathcal{T}^{\mathrm{W}}(\rho)+\mathcal{T}^{\mathrm{TF}}(\rho)-\mathcal{X}(\rho)+\mathcal{V}(\rho) .
$$

The first summand on the right is an inhomogeneity correction of the kinetic energy generalizing the Weizsäcker correction. Using the Fermi momentum $p(x):=$ $\left(3 \pi^{2} \rho(x)\right)^{1 / 3}$ it is

$$
\mathcal{T}^{\mathrm{W}}(\rho):=\int_{\mathbb{R}^{3}} \mathrm{~d} x \frac{3 \lambda}{8 \pi^{2}}(\nabla p(x))^{2} c f(p(x) / c)^{2}
$$

with $f(t)^{2}:=t\left(t^{2}+1\right)^{-\frac{1}{2}}+2 t^{2}\left(t^{2}+1\right)^{-1} \mathfrak{A} \mathfrak{r} \mathfrak{s i n}(t)$ where $\mathfrak{A} \mathfrak{r} \mathfrak{s i n}$ is the inverse function of the hyperbolic sine. The parameter $\lambda \in \mathbb{R}_{+}$is given by the gradient expansion as $1 / 9$ but is in the non-relativistic analogue sometimes taken as an adjustable parameter (Weizsäcker [31], Yonei and Tomishima [32], Lieb [21, 20]).

The second summand is the relativistic generalization of the Thomas-Fermi kinetic energy, namely

$$
\mathcal{T}^{\mathrm{TF}}(\rho):=\int_{\mathbb{R}^{3}} \mathrm{~d} x \frac{c^{5}}{8 \pi^{2}} T^{\mathrm{TF}}\left(\frac{p(x)}{c}\right)
$$

with $T^{\mathrm{TF}}(t):=t\left(t^{2}+1\right)^{3 / 2}+t^{3}\left(t^{2}+1\right)^{1 / 2}-\mathfrak{A} \mathfrak{r} \mathfrak{s i n}(t)-\frac{8}{3} t^{3}$.

The third summand is a relativistic generalization of the exchange energy. It is

$$
\mathcal{X}(\rho):=\int_{\mathbb{R}^{3}} \mathrm{~d} x \frac{c^{4}}{8 \pi^{3}} X\left(\frac{p(x)}{c}\right)
$$

with $X(t):=2 t^{4}-3\left[t\left(t^{2}+1\right)^{\frac{1}{2}}-\mathfrak{A} \mathfrak{r} \mathfrak{s i n}(t)\right]^{2}$.

Eventually, the last summand is the potential energy, namely the sum of the electron-nucleus and the electron-electron interaction. It is

$$
\mathcal{V}(\rho):=-Z \int_{\mathbb{R}^{3}} \mathrm{~d} x \rho(x)|x|^{-1}+\underbrace{\frac{1}{2} \int_{\mathbb{R}^{3}} \mathrm{~d} x \int_{\mathbb{R}^{3}} \mathrm{~d} y \rho(x) \rho(y)|x-y|^{-1}}_{=: \mathcal{D}[\rho]} .
$$

Using $F(t):=\int_{0}^{t} \mathrm{~d} s f(s)$, the functional $\mathcal{E}_{c, Z}^{\mathrm{TFWD}}$ is naturally defined on

$$
P:=\left\{\rho \in L^{\frac{4}{3}}\left(\mathbb{R}^{3}\right) \mid \rho \geq 0, \mathcal{D}[\rho]<\infty, F \circ p \in D^{1}\left(\mathbb{R}^{3}\right)\right\}
$$

and bounded from below [9] for all $c$ and $Z$. In fact Chen et al. [8] obtained a Thomas-Fermi type lower bound for fixed ratio $\kappa:=Z / c$. Unfortunately its Thomas-Fermi constant $\gamma_{e}$ is less than $\gamma_{\mathrm{TF}}:=\left(3 \pi^{2}\right)^{\frac{2}{3}}$, the correct physical value.

For comparison we need the non-relativistic Thomas-Fermi functional

$$
\mathcal{E}_{Z}^{\mathrm{TF}}(\rho):=\frac{3}{10} \gamma_{\mathrm{TF}} \int_{\mathbb{R}^{3}} \mathrm{~d} x \rho(x)^{\frac{5}{3}}+\mathcal{V}(\rho)
$$

defined on $I:=\left\{\rho \in L^{\frac{5}{3}}\left(\mathbb{R}^{3}\right) \mid \rho \geq 0, \mathcal{D}[\rho]<\infty\right\}$. The functional is bounded from below (Simon [27]) and its infimum fulfills the scaling relation

$$
E^{\mathrm{TF}}(Z):=\inf \mathcal{E}_{Z}^{\mathrm{TF}}(I)=-e^{\mathrm{TF}} Z^{\frac{7}{3}}
$$

where $e^{\mathrm{TF}}=-E^{\mathrm{TF}}(1)$ (Gombas [17], Lieb and Simon [23]). There exists a unique minimizer of $\mathcal{E}_{Z}^{\mathrm{TF}}$ which we denote by $\sigma$.

Our first result is 
Theorem 1. Assume $\kappa:=Z / c \in \mathbb{R}_{+}$fixed. Then, as $Z \rightarrow \infty$,

$$
\inf \mathcal{E}_{c, Z}^{\mathrm{TFWD}}(P)=-e^{\mathrm{TF}} Z^{\frac{7}{3}}+O\left(Z^{2}\right) .
$$

Our second result is the stability of the second kind of the functional which we address in Section 3.

From a mathematical perspective it might come as a surprise that Engel's and Dreizler's density functional - derived by purely formal methods from a quantum theory which is still lacking a full mathematical understanding - yields a fundamental feature like the ground state energy of heavy atoms to leading order quantitatively correct, in full agreement with the $N$-particle descriptions of heavy atoms like the Chandrasekhar Hamiltonian and no-pair Hamiltonians (Sørensen [26], [6], Solovej [28], Frank et al. [15, 16], [19]). It remains to be seen whether this is also true for other quantities like the density or whether the functional can be used as a tool to investigate relativistic many particle systems - like Thomas-Fermi theory in non-relativistic many body quantum mechanics - or, whether it even can shed light on a deeper understanding of quantum electrodynamics.

\section{Bounds on the EnERgy}

2.1. Upper Bound on the Energy. We begin with an innocent lemma.

Lemma 1. Assume $\rho: \mathbb{R}^{3} \rightarrow \mathbb{R}_{+}$such that $p:=\left(3 \pi^{2} \rho\right)^{\frac{1}{3}}$ has partial derivatives with respect to all variables at $x \in \mathbb{R}^{3}$. Then

$$
\frac{3}{8 \pi^{2}}|\nabla p(x)|^{2} c f(p(x) / c) \leq|(\nabla \sqrt{\rho})(x)|^{2} .
$$

Thus every nonnegative $\rho$ with $\nabla \sqrt{\rho} \in L^{2}\left(\mathbb{R}^{3}\right)$ fulfills

$$
\mathcal{T}^{\mathrm{W}}(\rho) \leq \lambda \int_{\mathbb{R}^{3}}|\nabla \sqrt{\rho}|^{2} .
$$

Proof. We set $\psi:=\sqrt{\rho}$ and compute

$$
\begin{aligned}
& \frac{3}{8 \pi^{2}}|\nabla p(x)|^{2} c f(p(x) / c) \\
= & \frac{3^{2}}{8}|\nabla \sqrt[3]{\rho}(x)|^{2}\left(\frac{\psi^{\frac{2}{3}}(x)}{\sqrt{1+(p(x) / c)^{2}}}+2 \frac{\psi^{\frac{2}{3}}(x)(p(x) / c) \mathfrak{A} \mathfrak{r} \mathfrak{s i n}(p(x) / c)}{1+(p(x) / c)^{2}}\right) \\
\leq & \frac{1}{2}|\nabla \psi(x)|^{2} \max \left\{\frac{\sqrt{1+t^{2}}+2 t \mathfrak{A} \mathfrak{r} \mathfrak{s i n}(t)}{1+t^{2}} \mid t \in \mathbb{R}_{+}\right\} \leq|\nabla \psi(x)|^{2} .
\end{aligned}
$$

Of course the illuminati are hardly impressed by (11), since dominating relativistic energies by non-relativistic ones is common place for them. Presumably not even use of the numerically correct value 1.658290113 of the maximum in the proof instead of the estimate 2 would change that.

Now we turn to the upper bound on the left side of (9). It will be practical to use the non-relativistic Thomas-Fermi-Weizsäcker functional

$$
\mathcal{E}_{Z}^{\mathrm{nrTFW}}(\rho):=\frac{\beta}{2} \int_{\mathbb{R}^{3}}|\nabla \sqrt{\rho}|^{2}+\mathcal{E}_{Z}^{\mathrm{TF}}(\rho)
$$

where $\beta \in \mathbb{R}_{+}$. It is defined on $J:=\left\{\rho \in L^{\frac{5}{3}}\left(\mathbb{R}^{3}\right) \mid \rho \geq 0, \sqrt{\rho} \in D^{1}\left(\mathbb{R}^{3}\right), \mathcal{D}[\rho]<\infty\right\}$, has a unique minimizer $\rho_{W}$ with $\int_{\mathbb{R}^{3}} \rho_{W} \leq Z+C$, and $\int_{\mathbb{R}^{3}} \rho_{W}^{\frac{5}{3}}=O\left(Z^{\frac{7}{3}}\right)$ (Benguria [1], Benguria et al. [2], Benguria and Lieb [3]). Moreover,

$$
E^{\mathrm{nrTFW}}(Z)=\mathcal{E}_{Z}^{\mathrm{nrTFW}}\left(\rho_{W}\right)=E^{\mathrm{TF}}(Z)+D_{\beta} Z^{2}+o\left(Z^{2}\right)
$$


for some $\beta$-dependent constant $D_{\beta} \in \mathbb{R}_{+}$(Lieb and Liberman [21] and Lieb [20, Formula (1.6)]).

In the following we pick $\beta=2$ and use the minimizer $\rho_{W}$ of the non-relativistic Thomas-Fermi-Weizsäcker functional as a test function.

We estimate the exchange term first. Since $-X(t) \leq t^{4}$, we get

$$
-\mathcal{X}\left(\rho_{W}\right) \leq \frac{\left(3 \pi^{2}\right)^{\frac{4}{3}}}{8 \pi^{3}} \int_{\mathbb{R}^{3}} \rho_{w}^{\frac{4}{3}} \leq C \sqrt{\int_{\mathbb{R}^{3}} \rho_{W}^{\frac{5}{3}} \int_{\mathbb{R}^{3}} \rho_{W}}=O\left(Z^{\frac{5}{3}}\right) .
$$

Thus, since $T^{\mathrm{TF}}(t) \leq \frac{3}{5} t^{5}$,

(16) $\inf \mathcal{E}_{c, Z}^{\mathrm{TFWD}}(P) \leq \mathcal{E}_{c, Z}^{\mathrm{TFWD}}\left(\rho_{W}\right) \leq \mathcal{E}_{Z}^{\mathrm{nrTFW}}\left(\rho_{W}\right)+O\left(Z^{\frac{5}{3}}\right)=E^{\mathrm{TF}}(Z)+O\left(Z^{2}\right)$

which concludes the proof of the upper bound.

2.2. Lower Bound on the Energy. We set $\mathrm{d} \xi:=\mathrm{d} \xi / h^{3}=\mathrm{d} \xi /(2 \pi)^{3}$. (Note that the rationalized Planck constant $\hbar$ equals one in Hartree units and, therefore, $h=2 \pi$.)

We introduce the notation $(a)_{-}:=\min \{0, a\}$ and write $\varphi_{\sigma}:=Z /|\cdot|-\sigma *|\cdot|^{-1}$ for the Thomas-Fermi potential of the minimizer $\sigma$. We start again with a little Lemma.

Lemma 2. Assume $\kappa=Z / c$ fixed. Then, as $Z \rightarrow \infty$,

$$
\int_{|x|>\frac{1}{Z}} \mathrm{~d} x \int_{\mathbb{R}^{3}} \mathrm{~d} \xi\left(\frac{\xi^{2}}{2}-\varphi_{\sigma}(x)\right)_{-}-\int_{|x|>\frac{1}{Z}} \mathrm{~d} x \int_{\mathbb{R}^{3}} \mathrm{~d} \xi\left(\sqrt{c^{2} \xi^{2}+c^{4}}-c^{2}-\varphi_{\sigma}(x)\right)_{-}=O\left(Z^{2}\right) .
$$

Again, it does not come as a surprise to the physicist that relativistic and nonrelativistic theory give the same result up to errors, if the innermost electrons, i.e., in particular the fast moving, are disregarded.

Proof. Since $\xi^{2} / 2 \geq \sqrt{c^{2} \xi^{2}+c^{4}}-c^{2}$, the left side of the claimed inequality cannot be negative. Thus, we merely need an upper bound:

$$
\begin{aligned}
& \int_{|x|>\frac{1}{Z}} \mathrm{~d} x \int_{\mathbb{R}^{3}} \mathrm{~d} \xi\left(\frac{\xi^{2}}{2}-\varphi_{\sigma}(x)\right)_{-}-\int_{|x|>\frac{1}{Z}} \mathrm{~d} x \int_{\mathbb{R}^{3}} \mathrm{~d} \xi\left(\sqrt{c^{2} \xi^{2}+c^{4}}-c^{2}-\varphi_{\sigma}(x)\right)_{-} \\
\leq & \int_{|x|>\frac{1}{Z}} \mathrm{~d} x\left\{c^{5} \int_{\frac{\xi^{2}}{2}<\frac{\varphi_{\sigma}(x)}{c^{2}}} \mathrm{~d} \xi\left[\frac{1}{2} \xi^{2}-\left(\sqrt{\xi^{2}+1}-1\right)\right]\right. \\
& \left.-\int_{\sqrt{c^{2} \xi^{2}+c^{4}}-c^{2}<\varphi_{\sigma}(x) \leq \xi^{2} / 2} \mathrm{~d} \xi\left(\sqrt{c^{2} \xi^{2}+c^{4}}-c^{2}-\varphi_{\sigma}(x)\right)\right\} \\
\leq & \left.c_{|x|>\frac{1}{Z}} \mathrm{~d} x \int_{\leq} \mathrm{d} \xi+\int_{\frac{\xi^{2}}{2}<\frac{\varphi_{\sigma}(x)}{c^{2}}} \mathrm{~d} \xi\right)\left[\frac{1}{2} \xi^{2}-\left(\sqrt{\xi^{2}+1}-1\right)\right] \\
\leq & \frac{c^{5}}{8} \int_{|x|>\frac{1}{Z}} \mathrm{~d} x\left(\int_{\frac{\xi^{2}}{2}<\frac{\varphi \sigma(x)}{c^{2}}} \mathrm{~d} \xi+\int_{\sqrt{\xi^{2}+1}-1<\frac{\varphi_{\sigma}(x)}{c^{2}} \leq \frac{\xi^{2}}{2}}\right) \\
\leq & \frac{c^{5}}{8} \int_{|x|>\frac{1}{Z}} \mathrm{~d} x \int_{\sqrt{\xi^{2}+1}-1<\frac{\varphi_{\sigma}(x)}{c^{2}} \leq \frac{\xi^{2}}{2}} \mathrm{~d} \xi \xi|\xi|^{4} \leq \frac{c^{2}}{8 \kappa^{3}} \int_{|x|>1} \mathrm{~d} x \int_{\sqrt{\xi^{2}+1}-1<\frac{\kappa^{2}}{|x|}} \mathrm{d} \xi|\xi|^{4}
\end{aligned}
$$

where we used $\varphi_{\sigma}(x) \leq Z /|x|$ in the last inequality. Moreover, the resulting last integral obviously exists and is independent of $Z$. Thus the left side of the claimed inequality is bounded from above by a constant depending only on $\kappa$ times $Z^{2}$ quod erat demonstrandum. 
We turn to the lower bound on the left side of (9) and follow initially [8]. In fact, apart from minor modifications, we copy the high density part and focus on the low density part. We pick any $\rho \in P$ and address the parts of the energy separately.

2.2.1. The Weizsäcker Energy. Since $F(t) \geq t \sqrt{\mathfrak{A} \mathfrak{r} \mathfrak{s i n}(t)} / 2$ (see [9, Formula (90)]), Hardy's inequality gives the lower bound

$$
\mathcal{T}^{\mathrm{W}}(\rho) \geq \frac{3 \lambda c}{2^{7} \pi^{2}} \int_{\mathbb{R}^{3}} \mathrm{~d} x \frac{p(x)^{2} \mathfrak{A} \mathfrak{r a i n}\left(\frac{p(x)}{c}\right)}{|x|^{2}}=\frac{3^{\frac{5}{3}} \lambda c}{2^{7} \pi^{\frac{2}{3}}} \underbrace{\int_{\mathbb{R}^{3}} \mathrm{~d} x \frac{\rho(x)^{\frac{2}{3}} \mathfrak{A} \mathfrak{r a i n}\left(\frac{p(x)}{c}\right)}{|x|^{2}}}_{=: \mathcal{H}(\rho)} .
$$

2.2.2. The Potential Energy. Since $\sigma$ is positive, we have $\varphi_{\sigma}(x) \leq Z /|x|$. Then

$$
\mathcal{V}(\rho)=-\int_{\mathbb{R}^{3}} \mathrm{~d} x \varphi_{\sigma}(x) \rho(x)-2 \mathcal{D}(\sigma, \rho)+\mathcal{D}[\rho] \geq-\int_{\mathbb{R}^{3}} \mathrm{~d} x \varphi_{\sigma}(x) \rho(x)-\mathcal{D}[\sigma] .
$$

Splitting the integrals at $s$, using (19), and Schwarz's inequality yields

$$
\begin{aligned}
\mathcal{V}(\rho) \geq & -\int_{p(x) / c<s} \mathrm{~d} x \varphi_{\sigma}(x) \rho(x) \\
& -Z \int_{p(x) / c \geq s} \mathrm{~d} x \frac{\rho(x)^{\frac{1}{3}}}{|x|} \mathfrak{A} \mathfrak{r} \mathfrak{s i n}\left(\frac{p(x)}{c}\right)^{\frac{1}{2}} \frac{\rho(x)^{\frac{2}{3}}}{\mathfrak{A} \mathfrak{r} \mathfrak{s i n}\left(\frac{p(x)}{c}\right)^{\frac{1}{2}}}-\mathcal{D}[\sigma] \\
\geq & -\frac{Z}{\mathfrak{A} \mathfrak{r} \mathfrak{s i n}(s)^{\frac{1}{2}}} \mathcal{H}(\rho)^{\frac{1}{2}} \mathcal{T}_{>}(\rho)^{\frac{1}{2}}-\int_{p(x) / c<s} \mathrm{~d} x \varphi_{\sigma}(x) \rho(x)-\mathcal{D}[\sigma]
\end{aligned}
$$

with $\mathcal{T}_{>}(\rho):=\int_{p(x) / c>s} \mathrm{~d} x \rho(x)^{\frac{4}{3}}$.

\subsubsection{The Thomas-Fermi Term. First, we note that}

$$
\mathbb{R}_{+} \rightarrow \mathbb{R}_{+}, t \mapsto T^{\mathrm{TF}}(t) / t^{4}
$$

is monotone increasing from 0 to 2 . Thus

$$
\begin{aligned}
& \mathcal{T}^{\mathrm{TF}}(\rho)=\int_{p(x) / c<s} \mathrm{~d} x \frac{c^{5}}{8 \pi^{2}} T^{\mathrm{TF}}\left(\frac{p(x)}{c}\right)+\int_{p(x) / c \geq s} \mathrm{~d} x \frac{c^{5}}{8 \pi^{2}} T^{\mathrm{TF}}\left(\frac{p(x)}{c}\right) \\
\geq & \int_{p(x) / c<s} \mathrm{~d} x \frac{c^{5}}{8 \pi^{2}} T^{\mathrm{TF}}\left(\frac{p(x)}{c}\right)+\int_{p(x) / c \geq s} \mathrm{~d} x \frac{T^{\mathrm{TF}}(s)}{s^{4}} \frac{3}{8}\left(3 \pi^{2}\right)^{\frac{1}{3}} c \rho(x)^{\frac{4}{3}} \\
= & \int_{p(x) / c<s} \mathrm{~d} x \frac{c^{5}}{8 \pi^{2}} T^{\mathrm{TF}}\left(\frac{p(x)}{c}\right)+\frac{3}{8} \frac{T^{\mathrm{TF}}(s)}{s^{4}} \gamma_{\mathrm{TF}^{\frac{1}{2}} c \mathcal{T}_{>}}(\rho) .
\end{aligned}
$$

2.2.4. Exchange Energy. Since $X$ is bounded from above and $X(t)=O\left(t^{4}\right)$ at $t=0$, we have that for every $\alpha \in[0,4]$ there is an $\eta_{0}$ such that $X(t) \leq \eta_{0} t^{\alpha}$. We pick $\alpha=3$ in which case $\xi_{0} \approx 1.15$. Thus, with $\eta:=\eta_{0} /(4 \pi) \approx 0.0914$, we have

$$
\mathcal{X}(\rho) \leq \frac{c \eta_{0}}{4 \pi} N=\eta c N
$$

2.2.5. The Total Energy. Adding everything up yields

$$
\begin{aligned}
\mathcal{E}_{c, Z}^{\mathrm{TFWD}}(\rho) \geq & \frac{3^{\frac{5}{3}} \lambda c}{2^{7} \pi^{\frac{2}{3}}} \mathcal{H}(\rho)+\frac{3}{8} \frac{T^{\mathrm{TF}}(s)}{s^{4}} \gamma_{\mathrm{TF}^{2}}{ }^{\frac{1}{2}} c \mathcal{T}_{>}(\rho)-\frac{Z}{\mathfrak{A} \mathfrak{r} \mathfrak{i n}(s)^{\frac{1}{2}}} \mathcal{H}(\rho)^{\frac{1}{2}} \mathcal{T}_{>}(\rho)^{\frac{1}{2}} \\
& \left.+\int_{\frac{p(x)}{c}<s} \mathrm{~d} x \frac{c^{5}}{8 \pi^{2}} T^{\mathrm{TF}}\left(\frac{p(x)}{c}\right)-\varphi_{\sigma}(x) \rho(x)\right)-\mathcal{D}[\sigma]-\xi c N .
\end{aligned}
$$

We pick $s \in \mathbb{R}_{+}$such that the sum of the first three summands on the right of (24) is a complete square, i.e., fulfilling

$$
\sqrt{\frac{3^{\frac{5}{3}}}{2^{7} \pi^{\frac{2}{3}}} \frac{3 T^{\mathrm{TF}}(s)\left(3 \pi^{2}\right)^{\frac{1}{3}}}{8 s^{4}}}=\frac{Z}{c \sqrt{\lambda}} \frac{1}{2 \mathfrak{A} \mathfrak{r} \mathfrak{s i n}(s)^{\frac{1}{2}}} .
$$


The solution is uniquely determined, because of (21) (and the line below) and $\mathfrak{A} \mathfrak{r} \mathfrak{s i n}(s)$ is also monotone increasing from 0 to $\infty$. Call the corresponding $s s_{0}$. Obviously, $s_{0}$ does not depend on $c$ and $Z$ independently but only on the ratio $\kappa:=Z / c$ and is strictly monotone increasing from 0 to $\infty$.

We set

$$
\begin{aligned}
I_{s, Z} & :=\left\{x \in \mathbb{R}^{3}|p(x) / c<s,| x \mid<1 / Z\right\}, \\
A_{s, Z} & :=\left\{x \in \mathbb{R}^{3}|p(x) / c<s,| x \mid \geq 1 / Z\right\} .
\end{aligned}
$$

Then

$$
\mathcal{E}_{c, Z}^{\mathrm{TFWD}}(\rho) \geq I+A-\mathcal{D}[\sigma]-\xi c N
$$

with

$$
\begin{aligned}
I & :=\int_{I_{s, Z}} \mathrm{~d} x\left(\frac{c^{5}}{8 \pi^{2}} T^{\mathrm{TF}}\left(\frac{p(x)}{c}\right)-\varphi_{\sigma}(x) \rho(x)\right) \\
A & :=\int_{A_{s, Z}} \mathrm{~d} x\left(\frac{c^{5}}{8 \pi^{2}} T^{\mathrm{TF}}\left(\frac{p(x)}{c}\right)-\varphi_{\sigma}(x) \rho(x)\right)
\end{aligned}
$$

We estimate $I$ from below by dropping the TF-term, using $\varphi_{\sigma}(x) \leq Z /|x|$, and observing that $x \in I_{s, Z}$. We get

$$
I \geq-C_{\kappa} Z c^{3} \int_{0}^{Z^{-1}} \mathrm{~d} r r=-C_{\kappa} Z^{2}
$$

where $C_{\kappa}$ is a generic constant depending on $\kappa$ only. In other words, we can pull the Coulomb tooth paying a negligible price.

Next we estimate $A$ from below by keeping $A_{s, Z}$ fixed and minimizing the integrand at each point $x \in A_{s, Z}$ by varying the values $p(x) \in \mathbb{R}_{+}$. We get

$$
\begin{aligned}
A & \geq 2 \int_{A_{s, Z}} \mathrm{~d} x \int_{\mathbb{R}^{3}} \mathrm{~d} \xi\left(\sqrt{c^{2} \xi^{2}+c^{4}}-c^{2}-\varphi_{\sigma}(x)\right)_{-} \\
& \geq 2 \int_{|x| \geq 1 / Z} \mathrm{~d} x \int_{\mathbb{R}^{3}} \mathrm{~d} \xi\left(\sqrt{c^{2} \xi^{2}+c^{4}}-c^{2}-\varphi_{\sigma}(x)\right)_{-} .
\end{aligned}
$$

Although at first glance the first inequality might seem abrupt, it is easily checked that the Thomas-Fermi functional (relativistic or non-relativistic, restricted to some region in space $M$ ) with kinetic energy $T(\xi)$ and external potential $\varphi$ is merely the marginal functional (integrating out the momentum variable $\xi$ ) of the phase space variational principle

$$
\mathcal{E}_{\Gamma}(\gamma):=\int_{M} \mathrm{~d} x \int_{\mathbb{R}^{3}} \mathrm{~d} \xi(T(\xi)-\varphi(x)) \gamma(x, \xi)
$$

with $\gamma\left(M, \mathbb{R}^{3}\right) \subset[0,2]$ and the choice $\gamma(x, \xi):=\chi_{\left\{(x, \xi) \in M \times \mathbb{R}^{3}|| \xi \mid<p(x)\right\}}$ for given Fermi momentum $p$. Eventually, since $\gamma$ has only values between 0 and 2, (32) is obviously minimized by the characteristic function of the support of the negative part of $T(\xi)-\varphi(x)$ times 2 .

Metaphorically speaking the second inequality of (31) states, that we can bound the energy from below by a toothless relativistic Thomas-Fermi energy.

Now, by Lemma 2 this equals the corresponding non-relativistic expression up to errors of order $O\left(Z^{2}\right)$, i.e., we have

$$
\begin{aligned}
\mathcal{E}_{c, Z}^{\mathrm{TFWD}}(\rho) & \geq 2 \int_{|x| \geq 1 / Z} \mathrm{~d} x \int_{\mathbb{R}^{3}} \mathrm{~d} \xi\left(\frac{1}{2} \xi^{2}-\varphi_{\sigma}(x)\right)_{-}-\mathcal{D}[\sigma]-C_{\kappa} Z^{2} \\
& \geq 2 \int_{\mathbb{R}^{3}} \mathrm{~d} x \int_{\mathbb{R}^{3}} \mathrm{~d} \xi\left(\frac{1}{2} \xi^{2}-\varphi_{\sigma}(x)\right)_{-}-\mathcal{D}[\sigma]-C_{\kappa} Z^{2} \\
& =\mathcal{E}_{Z}^{\mathrm{TF}}(\sigma)-C_{\kappa} Z^{2}=-e^{\mathrm{TF}} Z^{7 / 3}-C_{\kappa} Z^{2}
\end{aligned}
$$


which concludes the proof of the desired lower bound and therefore also the proof of $(9)$.

\section{Stability of Matter}

For several atoms the potential term $\mathcal{V}$ in (5) is replaced by

$$
\mathcal{V}(\rho):=-\sum_{k=1}^{K} \int_{\mathbb{R}^{3}} \mathrm{~d} x \frac{Z_{k} \rho(x)}{\left|x-R_{k}\right|}+\mathcal{D}[\rho]+\sum_{1 \leq k<l \leq K} \frac{Z_{k} Z_{l}}{\left|R_{k}-R_{l}\right|}
$$

with pairwise different positions of the nuclei $\mathfrak{R}:=\left(R_{1}, \ldots, R_{K}\right) \in \mathbb{R}^{3 K}$, and atomic numbers $\mathfrak{Z}:=\left(Z_{1}, \ldots, Z_{K}\right) \in \mathbb{R}_{+}^{K}$. The first term is the attraction potential between the electrons and the nuclei, the second term, the electron-electron interaction, is unchanged, and the third is the repulsion between the nuclei. We write $\mathcal{E}_{\mathfrak{R}, \mathfrak{Z}}^{\mathrm{TFW}}$ for the otherwise unchanged Engel-Dreizler functional. We are interested in finding a lower bound to $\mathcal{E}_{\mathfrak{R}, 3}^{\mathrm{TFWD}}$ which is uniform in the density $\rho$ as long as the constraint $\int \rho=N$ is respected, is uniform in $R_{1}, \ldots, R_{K}$ and linear in $N$ and $K$, i.e., we wish to show that the energy per particle is bounded from below by the same constant irrespective of the electron density and the positions of the nuclei. This property is also known as stability of the second kind, thermodynamic stability, and stability of matter.

Benguria et al. [4] showed stability of matter for the massless version of this functional without the regularizing $\mathfrak{A} \mathfrak{r s i n}$-term in $\mathcal{T}^{\mathrm{W}}$ and without the exchange correction.

Initially we restrict to the case of equal atomic numbers $Z$. We begin by extracting all Coulomb teeth which is conveniently done using [24, Formula (5.2)]: given disjoint balls $B_{1}, \ldots, B_{K}$ with centers at $R_{1}, \ldots, R_{K}$ and radii $D_{1}, \ldots, D_{K}$ we have

$$
-\Delta \geq \sum_{\kappa=1}^{K}\left(\frac{1}{4\left|\cdot-R_{\kappa}\right|^{2}}-D_{k}^{-2} Y\left(\frac{\left|\cdot-R_{\kappa}\right|}{D_{\kappa}}\right)\right)_{+} \chi_{B_{\kappa}}
$$

with $Y(r)=1+r^{2} / 4$. We also set $H_{k}(x):=2 \sqrt{Y\left(\left|x-R_{k}\right| / D_{K}\right)} / D_{k}$. We pick the radii maximal, namely $D_{k}$ as half the distance of the $k$-th nucleus to its nearest neighbor.

We also use Lieb and Yau's electrostatic inequality [24, Formula (4.5)]: we write $\Gamma_{1}, \ldots, \Gamma_{K}$ for the Voronoi cells of $R_{1}, \ldots, R_{K}$ and set

$$
\Phi(x):=\sum_{l=1}^{K} \Gamma_{l}(x) \sum_{k=1, k \neq l}^{K} \frac{Z}{\left|x-R_{k}\right|},
$$

i.e., the nuclear potential at point $x$ of all nuclei except the one from the cell in which $x$ lies. With this notation the electrostatic inequality reads

$$
\frac{1}{2} D[\nu]-Z \int_{\mathbb{R}^{3}} \Phi(x) \mathrm{d} \nu(x)+Z^{2} \sum_{1 \leq k<l \leq K} \frac{1}{\left|R_{k}-R_{l}\right|} \geq \frac{Z^{2}}{8} \sum_{k=1}^{K} \frac{1}{D_{k}}
$$

for any bounded measure and $Z \in \mathbb{R}_{+}$.

With these tools we modify the atomic lower bounds term by term.

3.1. The Weizsäcker Energy. Instead of (18) we get

$$
\mathcal{T}^{\mathrm{W}}(\rho) \geq \frac{3^{\frac{5}{3}} \lambda c}{2^{7} \pi^{\frac{2}{3}}} \sum_{k=1}^{K} \underbrace{\int_{B_{k}} \mathrm{~d} x \rho(x)^{\frac{2}{3}} \mathfrak{A} \mathfrak{r} \mathfrak{s i n}\left(\frac{p(x)}{c}\right)\left(\left|x-R_{k}\right|^{-2}-H_{k}(x)^{2}\right)_{+}}_{=: \mathcal{H}_{k}(\rho)}
$$

where we use (35) and $F(t) \geq t \sqrt{\mathfrak{A} \mathfrak{r i n}(s)} / 2$ (see [9, Formula (90)]). 
3.2. The Potential Energy. Using (37) and $\sqrt{\left(a^{2}-b^{2}\right)_{+}} \geq a-b$ for $a, b \in \mathbb{R}_{+}$ we get

$$
\begin{aligned}
& \mathcal{V}(\rho) \geq-\sum_{k=1}^{K}\left(\int_{B_{K}} \mathrm{~d} x+\int_{\Gamma_{k} \backslash B_{k}} \mathrm{~d} x\right) \frac{Z \rho(x)}{\left|x-R_{k}\right|}+\frac{Z^{2}}{8} \sum_{k=1}^{K} \frac{1}{D_{k}} \\
\geq & -\sum_{k=1}^{K} Z\left\{\int_{B_{k}, p(x) / c \geq s} \mathrm{~d} x \rho(x)\left[\sqrt{\left(\frac{1}{\left|x-R_{k}\right|^{2}}-H_{k}(x)^{2}\right)_{+}}+H_{k}(x)\right]\right. \\
& \left.+\int_{B_{k}, p(x) / c \leq s} \mathrm{~d} x \frac{\rho(x)}{\left|x-R_{k}\right|}+\int_{\Gamma_{k} \backslash B_{k}} \frac{\rho(x)}{\left|x-R_{k}\right|}-\frac{Z}{8} \frac{1}{D_{k}}\right\} \\
\geq & -\sum_{k=1}^{K}\left(\frac{Z}{\sqrt{\mathfrak{A} \mathfrak{r} \mathfrak{s i n}(s)}} \sqrt{\mathcal{H}_{R_{k}}(\rho) \mathcal{T}_{R_{k}}(\rho)}+Z \int_{B_{k}, p(x) / c \geq s} \mathrm{~d} x H_{k}(x) \rho(x)\right. \\
& \left.+\int_{B_{k}, p(x) / c \leq s} \mathrm{~d} x \frac{Z \rho(x)}{\left|x-R_{k}\right|}+\int_{\Gamma_{k} \backslash B_{k}} \mathrm{~d} x \frac{Z \rho(x)}{\left|x-R_{k}\right|}\right)+\frac{Z^{2}}{8} \sum_{k=1}^{K} \frac{1}{D_{k}}
\end{aligned}
$$

with $\mathcal{T}_{R_{k}}(\rho):=\int_{B_{k}, p(x) / c \geq s} \mathrm{~d} x \rho(x)^{\frac{4}{3}}$.

3.3. The Combined Thomas-Fermi and Exchange Terms. We use $T^{\mathrm{TF}}(t) \geq$ $2 t^{4}-8 t^{3} / 3,(23)$, and set $\delta:=\left(3 \pi^{2}\right)^{\frac{1}{3}}$. This yields

$$
\mathcal{T}^{\mathrm{TF}}(\rho)-\mathcal{X}(\rho)=\int_{\mathbb{R}^{3}} \mathrm{~d} x \frac{c^{5}}{8 \pi^{2}} T^{\mathrm{TF}}\left(\frac{p(x)}{c}\right)-\eta c N \geq \frac{3}{4} \delta c \int_{\mathbb{R}^{3}} \mathrm{~d} x \rho(x)^{\frac{4}{3}}-C_{c} N,
$$

3.4. The Total Energy. Adding again all up yields

$$
\begin{aligned}
& \quad \mathcal{E}_{c, Z}^{\mathrm{TFWD}}(\rho) \\
& \geq \sum_{k=1}^{K} c\left[\frac{3^{\frac{5}{3}} \lambda}{2^{7} \pi^{\frac{2}{3}}} \mathcal{H}_{R_{k}}(\rho)+\frac{3}{8} \delta \mathcal{T}_{R_{k}}(\rho)-\frac{\kappa}{\sqrt{\mathfrak{A} \mathfrak{r} \mathfrak{s i n}(s)}} \sqrt{\mathcal{H}_{R_{k}}(\rho) \mathcal{T}_{R_{k}}(\rho)}\right. \\
& \quad+\int_{B_{k}, \frac{p(x)}{c}<s} \mathrm{~d} x\left(\frac{3}{4} \delta \rho(x)^{\frac{4}{3}}-\frac{\kappa \rho(x)}{\left|x-R_{k}\right|}\right) \\
& \quad+\int_{B_{k}, p(x) / c>s} \mathrm{~d} x\left(\frac{3}{8} \delta \rho(x)^{\frac{4}{3}}-\kappa H_{k}(x) \rho(x)\right) \\
& \left.\quad+\int_{\Gamma_{k} \backslash B_{k}} \mathrm{~d} x\left(\frac{3}{4} \delta \rho(x)^{\frac{4}{3}}-\frac{\kappa \rho(x)}{\left|x-R_{k}\right|}\right)\right] \\
& \quad+\sum_{k=1}^{K} \frac{Z^{2}}{8 D_{k}}-C_{c} N .
\end{aligned}
$$

We pick $s$ such that

$$
2 \sqrt{\frac{3^{\frac{5}{3}} \lambda}{2^{7} \pi^{\frac{2}{3}}} \frac{3}{8}} \delta=\frac{\kappa}{\sqrt{\mathfrak{A} \mathfrak{r i n}(s)}}
$$

which makes (42) a sum of complete squares. Next

$$
\begin{gathered}
(43) \geq \delta(c s)^{4} \inf \left\{\int_{p(x)<1} \mathrm{~d} x\left(\frac{3}{4} \rho(x)^{\frac{4}{3}}-\frac{\kappa \rho(x)}{c s \delta|x|}\right) \mid \rho \in P\right\} \\
\geq \frac{c s \kappa^{3}}{\delta^{2}} \inf \left\{\int_{p(x)<1} \mathrm{~d} x\left(\frac{3}{4} \rho(x)^{\frac{4}{3}}-\frac{\rho(x)}{|x|}\right) \mid \rho \in P\right\} \geq-C \frac{c s \kappa^{3}}{\delta^{2}}
\end{gathered}
$$


where we replaced $p$ by $c s p$ in the first step and $x$ by $\kappa /(\operatorname{cs} \delta) x$ in the second step. Thus (48) yields, after summation, $K$ times a constant which is irrelevant for stability. Furthermore,

$$
(44) \geq-\frac{2^{4} \kappa^{4} \cdot 4 \pi}{2 \cdot 4 \delta^{3} D_{k}} \int_{0}^{1} \mathrm{~d} r r^{2} 2^{4}\left(1+r^{2} / 4\right)^{2}=-\frac{5944 \pi \kappa^{4}}{105 \delta^{3} D_{k}}
$$

and using $\int_{\Gamma_{k} \backslash B_{k}} \mathrm{~d} x\left|x-R_{k}\right|^{-4} \leq 3 \pi / D_{k}$ (Lieb et al. [22, Formula (4.6)]) we get

$$
(45) \geq-\frac{\kappa^{4}}{4 \delta^{3}} \int_{\Gamma_{k} \backslash B_{k}} \frac{1}{\left|x-R_{k}\right|^{4}} \geq-\frac{3 \pi \kappa^{4}}{4 \delta^{3} D_{k}} .
$$

Thus, the energy per particle is bounded from below uniformly in $\rho, K$, and $N$, if

$$
c\left(\frac{2972 \pi \kappa^{4}}{105 \delta^{3}}+\frac{3 \pi \kappa^{4}}{4 \delta^{3}}\right) \leq \frac{Z^{2}}{8}
$$

i.e.,

$$
Z \leq Z_{\max }:=3 \frac{\sqrt{1686370 \pi}}{48182} c^{\frac{3}{2}} .
$$

Numerically, using the physical value of the velocity of light $c=137.037$ (Bethe and Salpeter [5, p. 84]), we get

$$
Z_{\max } \approx 229.9029615
$$

covering liberally all known elements. The result can be condensed into

Theorem 2. There exists a constant $C$ such that for all $\rho \in P$ and all pairwise different $R_{1}, \ldots, R_{K} \in \mathbb{R}^{3}$ and $Z_{1}=\ldots=Z_{K} \in\left[0, Z_{\max }\right]$

$$
\mathcal{E}_{\mathfrak{R}, \mathfrak{Z}}^{\mathrm{TFWD}}(\rho) \geq C \cdot(K+N) .
$$

We conclude with two remarks:

1. Theorem 2 holds actually for all $\mathfrak{Z} \in\left[0, Z_{\max }\right]^{K}$. Our proof obviously generalizes to that case, since the potential estimate (37) also generalizes in the obvious way.

2. It might be surprising that there is no requirement on a minimal velocity of light which is independent of the value of $Z$. This is different from other unrenormalized relativistic models like the Thomas-Fermi functional with inhomogeneity correction $(\sqrt{\rho},|\nabla| \sqrt{\rho})$ investigated by Lieb et al. [22, Formula (2.7)]. There we were forced to control the exchange energy by the Thomas-Fermi term. Here, this is no longer necessary: due to the renormalization in Engel's and Dreizler's derivation the exchange energy is bounded from below by a multiple of the particle number.

\section{ACKNOWLEDGMENTS}

Special thanks go to Rupert Frank for many inspiring discussions, in particular for pointing out that in addition to separation in high and low density regimes, a localization in space near the nucleus could be useful, and for critical reading of a substantial part of the manuscript.

Thanks go also to Hongshuo Chen for critical reading of the manuscript.

Partial support by the Deutsche Forschungsgemeinschaft (DFG, German Research Foundation) through Germany's Excellence Strategy EXC - 2111 - 390814868 is gratefully acknowledged. 


\section{REFERENCES}

[1] Rafael Benguria. The von Weizsäcker and Exchange Corrections in the Thomas-Fermi Theory. PhD thesis, Princeton, Department of Physics, June 1979.

[2] Rafael Benguria, Haim Brezis, and Elliott H. Lieb. The Thomas-Fermi-von Weizsäcker theory of atoms and molecules. Comm. Math. Phys., 79(2):167-180, 1981.

[3] Rafael Benguria and Elliott H. Lieb. The most negative ion in the Thomas-Fermi-von Weizsäcker theory of atoms and molecules. J. Phys. B., 18:1045-1059, 1985.

[4] Rafael D Benguria, Michael Loss, and Heinz Siedentop. Stability of atoms and molecules in an ultrarelativistic Thomas-Fermi-Weizsäcker model. Journal of Mathematical Physics, 49(1):012302, 2008.

[5] Hans A. Bethe and Edwin E. Salpeter. Quantum mechanics of one- and two-electron atoms. In S. Flügge, editor, Handbuch der Physik, XXXV, pages 88-436. Springer, Berlin, 1 edition, 1957.

[6] Roch Cassanas and Heinz Siedentop. The ground-state energy of heavy atoms according to Brown and Ravenhall: Absence of relativistic effects in leading order. J. Phys. A, 39(33):10405-10414, 2006.

[7] Subramanyan Chandrasekhar. The maximum mass of ideal white dwarfs. Astrophys. J., 74:81-82, 1931.

[8] Hongshuo Chen, Rupert L. Frank, and Heinz Siedentop. A statistical theory of heavy atoms: Energy and excess charge. arxiv:2010.12074, October 2020.

[9] Hongshuo Chen and Heinz Siedentop. On the excess charge of a relativistic statistical model of molecules with an inhomogeneity correction. Journal of Physics A: Mathematical and Theoretical, 53(39):395201, September 2020.

[10] T. Ekholm and R. L. Frank. On Lieb-Thirring inequalities for Schrödinger operators with virtual level. Comm. Math. Phys., 264(3):725-740, 2006.

[11] E. Engel and R. M. Dreizler. Field-theoretical approach to a relativistic Thomas-Fermi-DiracWeizsäcker model. Phys. Rev. A, 35:3607-3618, May 1987.

[12] E. Fermi. Un metodo statistico per la determinazione di alcune proprietá dell'atomo. Atti della Reale Accademia Nazionale dei Lincei, Rendiconti, Classe di Scienze Fisiche, Matematiche e Naturali, 6(12):602-607, 1927.

[13] E. Fermi. Eine statistische Methode zur Bestimmung einiger Eigenschaften des Atoms und ihre Anwendung auf die Theorie des periodischen Systems der Elemente. Z. Phys., 48:73-79, 1928.

[14] Rupert L. Frank, Elliott H. Lieb, and Robert Seiringer. Stability of relativistic matter with magnetic fields for nuclear charges up to the critical value. Comm. Math. Phys., 275(2):479489, 2007.

[15] Rupert L. Frank, Heinz Siedentop, and Simone Warzel. The ground state energy of heavy atoms: Relativistic lowering of the leading energy correction. Comm. Math. Phys., 278(2):549-566, 2008.

[16] Rupert L. Frank, Heinz Siedentop, and Simone Warzel. The energy of heavy atoms according to Brown and Ravenhall: the Scott correction. Doc. Math., 14:463-516, 2009.

[17] P. Gombás. Die statistische Theorie des Atoms und ihre Anwendungen. Springer-Verlag, Wien, 1 edition, 1949.

[18] P. Gombás. Statistische Behandlung des Atoms. In S. Flügge, editor, Handbuch der Pysik Atome II, volume 36, pages 109-231. Springer-Verlag, Berlin, 1956.

[19] Michael Handrek and Heinz Siedentop. On the maximal excess charge of the ChandrasekharCoulomb Hamiltonian in two dimension. Lett. Math. Phys., 103(8):843-849, 2013.

[20] Elliott H. Lieb. Analysis of the Thomas-Fermi-von Weizsäcker equation for an infinite atom without electron repulsion. Comm. Math. Phys., 85(1):15-25, 1982.

[21] Elliott H. Lieb and David A. Liberman. Numerical calculation of the Thomas-Fermi-von Weizsäcker function for an infinite atom without electron repulsion. Technical Report LA9186-MS, Los Alamos National Laboratory, Los Alamos, New Mexico, April 1982.

[22] Elliott H. Lieb, Michael Loss, and Heinz Siedentop. Stability of relativistic matter via Thomas-Fermi theory. Helv. Phys. Acta, 69(5/6):974-984, December 1996.

[23] Elliott H. Lieb and Barry Simon. The Thomas-Fermi theory of atoms, molecules and solids. Advances in Math., 23(1):22-116, 1977.

[24] Elliott H. Lieb and Horng-Tzer Yau. The stability and instability of relativistic matter. Comm. Math. Phys., 118:177-213, 1988.

[25] N. H. March and W. H. Young. Variational methods based on the density matrix. Proc. Phys. Soc., 72:182-192, 1958.

[26] Thomas Østergaard Sørensen. The large- $Z$ behavior of pseudorelativistic atoms. J. Math Phys., 46(5):052307, 24, 2005 
[27] Barry Simon. Functional Integration and Quantum Physics. Academic Press Inc. [Harcourt Brace Jovanovich Publishers], New York, 1979.

[28] Jan Philip Solovej, Thomas Østergaard Sørensen, and Wolfgang L. Spitzer. The relativistic Scott correction for atoms and molecules. Commun. Pure Appl. Math., 63:39-118, January 2010.

[29] L. H. Thomas. The calculation of atomic fields. Proc. Camb. Phil. Soc., 23:542-548, 1927.

[30] Yasuo Tomishima. A Relativistic Thomas-Fermi Theory. Progress of Theoretical Physics, 42(3):437-447, 091969.

[31] C. F. v. Weizsäcker. Zur Theorie der Kernmassen. Z. Phys., 96:431-458, 1935.

[32] Katsumi Yonei and Yasuo Tomishima. On the Weizsäcker correction to the Thomas-Fermi theory of the atom. Journal of the Physical Society of Japan, 20(6):1051-1057, 1965.

Mathematisches Institut, Ludwig-Maximilans Universität München, Theresienstrasse 39, 80333 München, Germany, and Munich Center for Quantum Science and Technology (MCQST), Schellingstr. 4, 80799 München, Germany

Email address: h.s@lmu.de 